\title{
SALT's venture into near infrared astronomy with RSS-NIR
}

\author{
Marsha J. Wolf ${ }^{\star}$ \\ University of Wisconsin - Madison \\ Astronomy Department, 475 N. Charter Street, Madison, WI, 53706, USA \\ E-mail: mwolfeastro.wisc.edu
}

The Robert Stobie Spectrograph (RSS) on SALT provides a versatile range of observing modes including single or multi-object, medium resolution spectroscopy, imaging, Fabry-Perot spectral imaging, and polarimetry, all over an 8 arcminute field of view covering the wavelength range of $\lambda=$ $320-1000 \mathrm{~nm}$. In 2018 we anticipate the addition of RSS-NIR, which will extend SALT's operational capability through $\lambda=1700 \mathrm{~nm}$. The near infrared arm was designed to replicate all the RSS operating modes and to be mated to RSS via a dichroic beamsplitter, allowing simultaneous observations in all modes over the entire $320-1700 \mathrm{~nm}$ range. Initially, however, RSS-NIR will be installed in the spectrometer room and fed with a fiber integral field unit (IFU). In this paper we discuss the changes this implies for operational modes, present a preliminary design of the new IFU, and motivate near infrared science that this instrument will enable on SALT.

SALT Science Conference 2015 -SSC2015-

1-5 June, 2015

Stellenbosch Institute of Advanced Study, South Africa

*Speaker.

(C) Copyright owned by the author(s) under the terms of the Creative Commons 


\section{Background and introduction}

The Robert Stobie Spectrograph (RSS) [1] is a versatile prime focus instrument on SALT. It provides observing modes that include single or multi-object, medium resolution spectroscopy, imaging, Fabry-Perot spectral imaging, and polarimetry, all over an 8 arcmin field of view and covering the wavelength range of $\lambda=320-1000 \mathrm{~nm}$. RSS was always envisioned to have a future near infrared arm that would extend capabilities through $\lambda=1700 \mathrm{~nm}$. This instrument, RSS-NIR [2], is being developed at the University of Wisconsin - Madison (PI: Marsha Wolf). A schematic of the combined prime focus instrument is shown in Figure 1. The telescope image plane is at the bottom. Light travels up through the collimator to a fold mirror that directs the light to the right into the existing RSS-VIS instrument. The fold mirror will be replaced with a dichroic beamsplitter that reflects visible light and transmits near infrared light up into RSS-NIR, with the wavelength split at $\lambda=900 \mathrm{~nm}$. To decrease the instrumental thermal background seen by the NIR detector, components beyond the beamsplitter are enclosed in an insulated enclosure that is cooled to $-40{ }^{\circ} \mathrm{C}$. Within that enclosure, the pre-dewar, is a cryogenic dewar operating at $120 \mathrm{~K}$ that houses the detector and final camera optics.

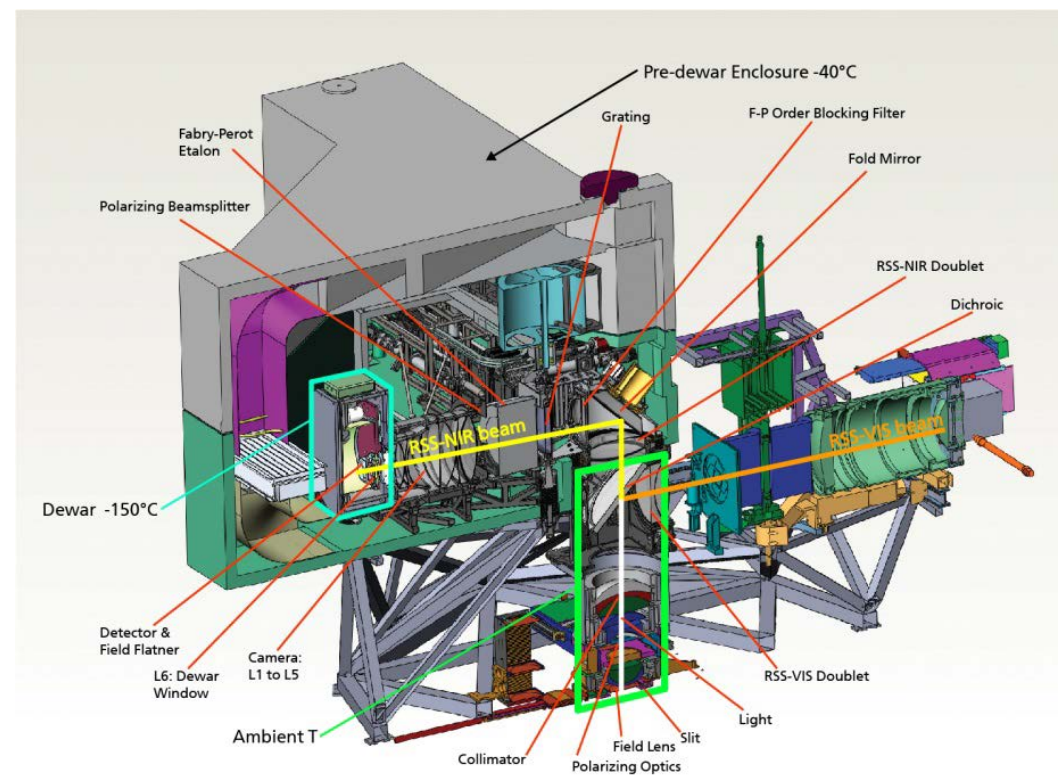

Figure 1. Schematic of the combined RSS-VIS-NIR instrument at prime focus.

Even with the cooled pre-dewar enclosure, the RSS-NIR detector sees thermal emission from the ambient temperature collimator optics and slitmask, limiting its long wavelength extent to $\lambda \sim 1700 \mathrm{~nm}$ for bright photon-limited objects and to $\lambda \sim 1550 \mathrm{~nm}$ for faint sky-limited objects. Upgrade options include a slit cooling system and long wavelength cutoff filters that would extend sky-limited operation out to $\lambda \sim 1670 \mathrm{~nm}$. The first instrument modes were to include spectroscopy (with only one of the planned suite of five gratings) and imaging, with 
Fabry-Perot and polarimetry modes left as future upgrade paths, although all mechanisms required to support these modes are built into the instrument.

In May 2015 the PI and the SALT Board made the decision to initially not mate RSS-NIR to RSS-VIS at prime focus, but to install it in the spectrometer room, fed by a fiber integral field unit (IFU). This decision was motivated by the needs to: 1) maximize current SALT science productivity, 2) minimize telescope downtime during instrument integration, 3) simplify future instrument maintenance, and 4) lower instrument performance risks. The throughput of RSSVIS was recently significantly increased by maintenance that involved removing it from the telescope, cleaning the optics, and replacing the lens coupling fluid that degrades over time. It is expected that RSS-VIS will require similar annual maintenance that would be severely complicated by the addition of RSS-NIR. The integration of the NIR arm into RSS, originally planned for 2016, would have required that RSS-VIS be off the telescope for a period of $2-3$ months. Due to its recent increase in performance and science productivity, the strategic decision was made to postpone the integration of RSS-NIR. Instead, a fiber cable and separate collimator will be built for RSS-NIR, which will now be housed in a cooled room below the telescope. No permanent changes will be made to RSS-NIR that would prevent its future installation at prime focus at a time when additional resources and funding are available.

\section{RSS-NIR capabilities}

\subsection{Observing modes}

A comparison of the RSS-NIR observing mode capabilities in the two different installation configurations (prime focus or spectrometer room) is summarized in Table 1. The largest performance hits incurred by fiber feeding RSS-NIR are the loss of the broad wavelength coverage afforded by simultaneous operation with RSS-VIS and the loss of upgrade paths to imaging, Fabry-Perot, and polarimetry observing modes. However, we gain performance in other areas, the first of which is the addition of an integral field spectroscopy observing mode. Furthermore, we gain performance with improved sky-subtraction due to fiber scrambling of SALT's moving pupil (see § 3) and lower instrumental thermal background. RSS-NIR will now have its own collimator with coatings optimized for the NIR spectral band and it will be cooled to $-40{ }^{\circ} \mathrm{C}$ with the rest of the instrument. Our initial estimates show that this lowered background will allow sky-limited spectroscopy on faint objects all the way out to $\lambda \sim 1700 \mathrm{~nm}$.

\subsection{Spectroscopy}

Spectroscopy on RSS-NIR is achieved through high efficiency volume phase holographic (VPH) diffraction gratings and an articulating camera to provide a very flexible array of observing setups. Predicted performance of the designed grating suite is shown in Figure 2 for a 1 arcsec slit width (left). Only the $950 \mathrm{l} / \mathrm{mm}$ grating (blue contours) will be initially available, but future gratings can be easily added. The right panel of Figure 2 shows the effect of fiber size on spectral resolution in the new fiber fed configuration. The baseline design fiber size is 1.33 arcsec, which will give a maximum spectral resolution of $\mathrm{R} \sim 6200$. 


\begin{tabular}{|l|l|}
\hline Mated to RSS-VIS at prime focus & Fiber fed in spectrograph room \\
\hline$\lambda=320-900 \mathrm{~nm}$ (VIS) $\& \lambda=900-1700 \mathrm{~nm}$ (NIR) & $\lambda=800-1700 \mathrm{~nm}$ \\
\hline Imaging (but no broadband filters) & No imaging \\
\hline Longslit or multi-object spectroscopy & Integral field spectroscopy \\
\hline 8 arcmin field of view & 24 x 28 arcsec field of view \\
\hline $\begin{array}{l}\text { Faint objects: sky-limited to } \lambda=1550 \mathrm{~nm} \\
\text { Bright objects: photon-limited to } \lambda=1700 \mathrm{~nm}\end{array}$ & Faint objects: sky-limited to $\lambda=1700 \mathrm{~nm}$ \\
\hline Potential upgrades: & NO upgrade path to imaging \\
\hline Broadband filters for imaging & NO upgrade path to polarimetry \\
\hline Polarimetry & NO upgrade path to Fabry-Perot \\
\hline Fabry-Perot & $\begin{array}{l}\text { Fiber positioner for multiple objects in } 8 \\
\text { arcmin field of view (requires new fiber cable) }\end{array}$ \\
\hline Cooled slit for sky-limited to $1670 \mathrm{~nm}$
\end{tabular}

Table 1. Comparison of RSS-NIR observing mode capabilities in the two installation configurations: mated to RSS-VIS at prime focus (left) and fed with an IFU in the spectrometer room (right).
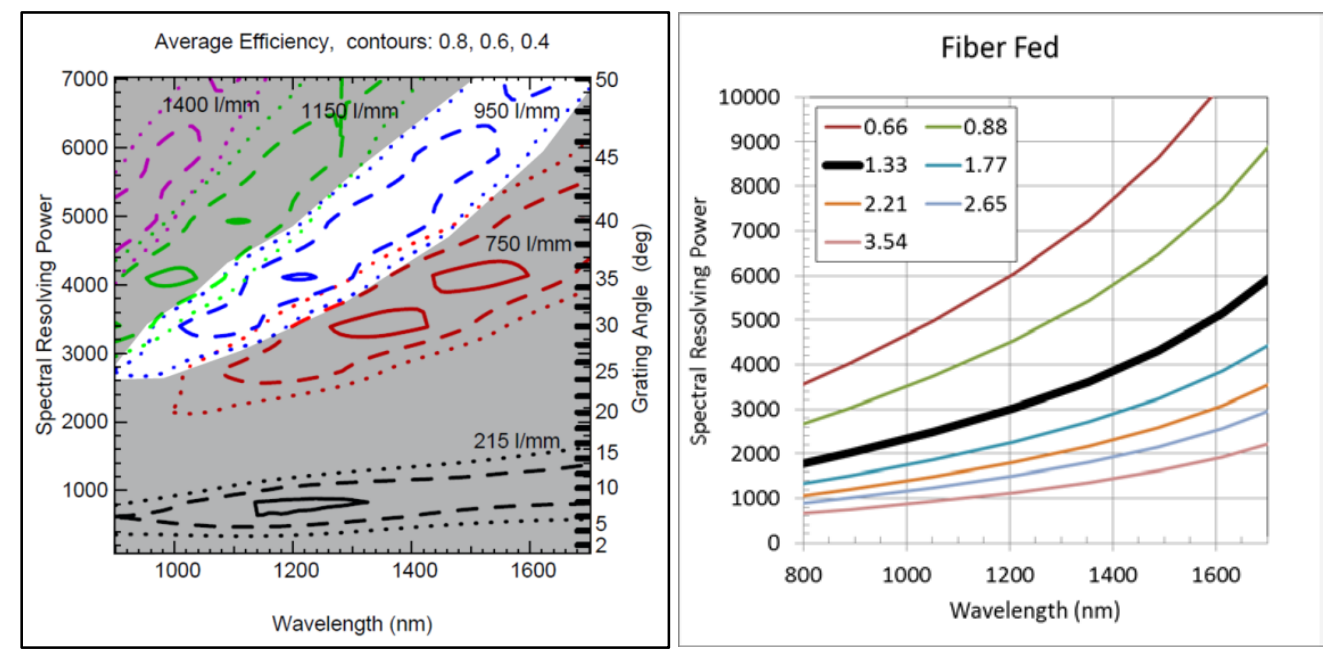

Figure 2. Predicted performance of the RSS-NIR VPH gratings. Left: The entire designed suite of gratings for RSS-NIR. Diffraction efficiency contours are 80\% (solid), 60\% (dashed), and 40\% (dotted). Since only the $950 \mathrm{l} / \mathrm{mm}$ grating will be delivered with the instrument, the rest of operational parameter space is greyed out. The spectral resolving power (R) is shown as a function of grating rotation angle (right axis) and wavelength for a 1 arcsec slit. Right: Spectral resolution as a function of central wavelength for different fiber sizes (arcsec subtended on-sky). The baseline design fiber size is 1.33 arcsec, shown as the thick black line.

\subsection{The integral field unit}

The fibers form the spectrograph pseudo-slit, thus the fiber diameter becomes the slit width. The decision on fiber size is a tradeoff between (i) small fibers for high spectral resolution but low grasp (A $\Omega$, where A is fiber area and $\Omega$ is fiber solid angle on sky) and (ii) large fibers with lower spectral resolution but high grasp for observing low surface brightness regions. Figure 2 shows that small 0.66 arcsec fibers could achieve spectral resolution up to $\mathrm{R} \sim$ 
10,000, but typical exposure times (maximum of $~ 10$ minutes due to NIR sky variability) would be read noise limited rather than sky-limited. For our preliminary baseline IFU design we have selected larger, 1.33 arcsec (300 micron core) fibers for the following reasons: 1) exposures will be photon-limited, 2) higher grasp fibers will take advantage of the fact that SALT excels at low surface brightness observations, 3 ) the size is more closely matched to the site seeing, and 4) larger fibers result in larger IFU field coverage on sky.

The number of fibers in the IFU head is limited by how many fibers fit along the 8 arcmin slit that feeds the spectrograph. This number is set by both the fiber size and the spacing between fibers to minimize crosstalk, given the point spread function (psf) of the instrument. Since the delivered image quality is affected by the collimator, the IFU design must iterate with the collimator design. For a start, we have adopted a pseudo-slit fiber spacing of 406 microns (2 pixel gap from edge to edge of fiber cores), similar to that used for the SDSS-IV MaNGA survey [3]. This gives a total of 247 fibers, 217 in the IFU head and two sets of 15 sky fibers. The baseline IFU geometry is shown in Figure 3.

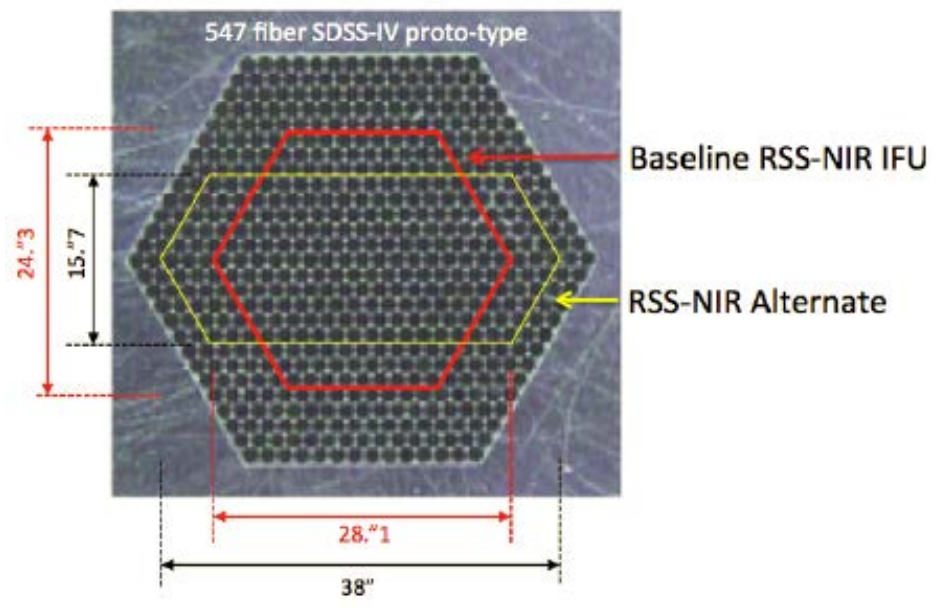

Figure 3. Baseline IFU geometry for RSS-NIR. The background image is the face of a 547 fiber IFU built by Matt Bershady (UW-Madison) and collaborators. The red and yellow hexagonal outlines show two options for the geometry of the RSS-NIR IFU. Its construction will use successful techniques proven on the SDSS-IV MaNGA IFUs and the larger prototype IFU pictured here.

\section{The advantage of fiber scrambling}

A fiber fed RSS-NIR will enable better flat-fielding and sky-subtraction than is currently possible with slit spectroscopy. Flat-fielding issues come from the fact that the fixed elevation of SALT requires the instrument payload to track across the primary mirror during observations, resulting in a variable pupil illumination with tracker position and field position in the focal plane, as shown in Figure 4. This introduces difficulties in flat fielding that, in principle, should be solved by the moving baffle system that mimics the variable pupil during flat-field lamp observations. In practice, this calibration system fails to reproduce the source illumination in both the near-field (i.e., the vignetting function) and far-field (the pupil illumination). This has disastrous implications for sky-subtraction and flux calibration of extended sources, even those 
that do not fill the slit in spectroscopic observations. Currently the only recourse is to take sky flats over identical tracks, which has proven impractical to execute and prohibitively inefficient. The problem will be even worse in the near infrared where the sky is significantly brighter, more variable, and filled with $\mathrm{OH}-$ emission lines.
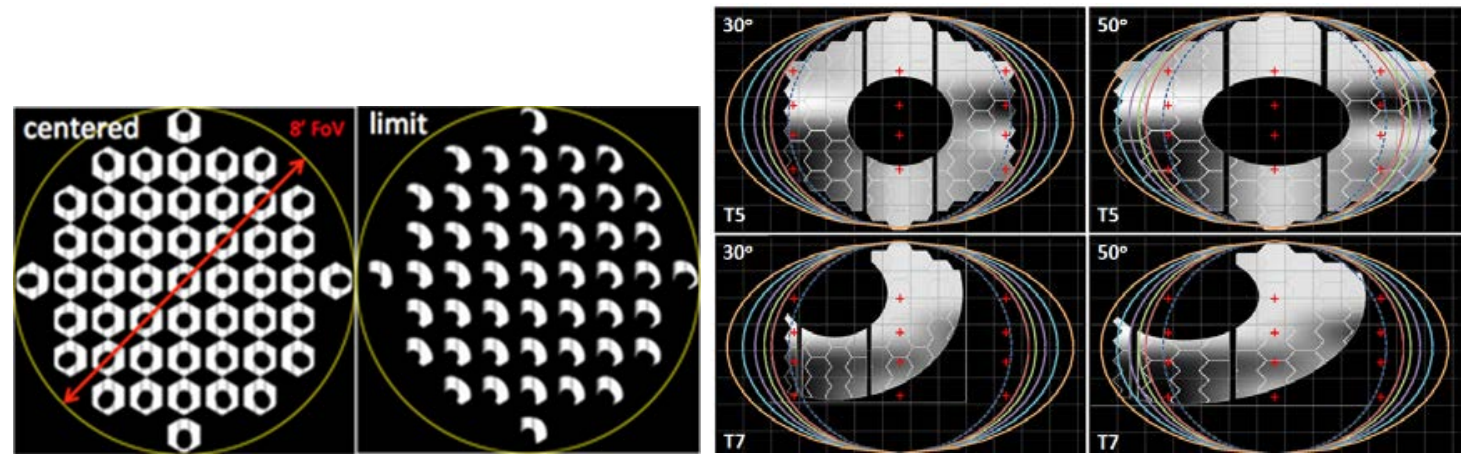

Figure 4. Left: Pupil illumination as function of focal-plane field location for centered and limiting tracker positions for SALT. Right: RSS pupil illumination of a VPH grating at 2 different grating angles (left $30^{\circ}$ and right $50^{\circ}$ ) with the tracker at the center (top) and limiting (bottom) positions. The greyscale inside the illuminated portions represents the underlying grating diffraction efficiency, measured at the locations of the red crosses and extrapolated to a surface.

The variable pupil creates further consequences for sky-subtraction because VPH gratings are known have wavelength-dependent spatial non-uniformities in diffraction efficiency across their clear aperture (likely due to variations in the DCG thickness). When different regions of the grating are illuminated by the changing pupil shape, the object's spectrum is diffracted with the different efficiency in that region, causing spectral features (sky lines) to change in intensity. The righthand panel in Figure 4 quantifies this point: each rectangle represents the grating; colored ellipses show the extent of a filled pupil for grating rotation angles from $0^{\circ}$ to $50^{\circ}$ if the beam were filled, while the shaded areas show the actual pupil illumination at two track extrema. Red crosses mark locations where performance data have been measured for the RSSNIR $950 \mathrm{l} / \mathrm{mm}$ grating and maps of this efficiency are indicated by the greyscale within the illiminated areas. Resulting wavelength-dependent diffraction efficiency variations of up to $12 \%$ are predicted from different tracker positions during a typical track. Similar effects, albeit at 5 6 times lower amplitude, are seen at different field positions in a given tracker location.

Figure 5 shows laboratory measurements of fiber output as a function of the input light distribution (telescope pupil shape) and the spot position on the fiber face. No matter the shape of the input pupil, the output is an azimuthally scrambled ring whose radius is related to the input spot position. To predict fiber performance on SALT, we constructed scrambled pupil images by forming annular averages of simulated SALT pupil images convolved with the spatial grating efficiency, as shown in Figure 6. Each individual ring was then smoothed by a radially-dependent Gaussian width [4], based on laboratory measurements, and then all smoothed rings were summed to produce the final azimuthally scrambled image. The effectiveness of sky subtraction was then evaluated by using "observed sky" from either (a) other tracker positions (going off target to observe a blank sky field) or (b) other field points 
along the slit for a given tracker position (taking sky along the slit off the target). The simulation results indicate that circular fibers, because of the stabalized pupil illumination of the grating, improve fractional sky residuals relative to longslit observations by a factor of $30-500\left(4 \times 10^{-4}\right.$ fractional sky residual, depending on grating angles and exact tracker positions). This means that even if near-field vignetting of the SALT focal-plane is not well corrected via dome flats, fibers should allow RSS-NIR to achieve nearly photon-limited sky subtraction.

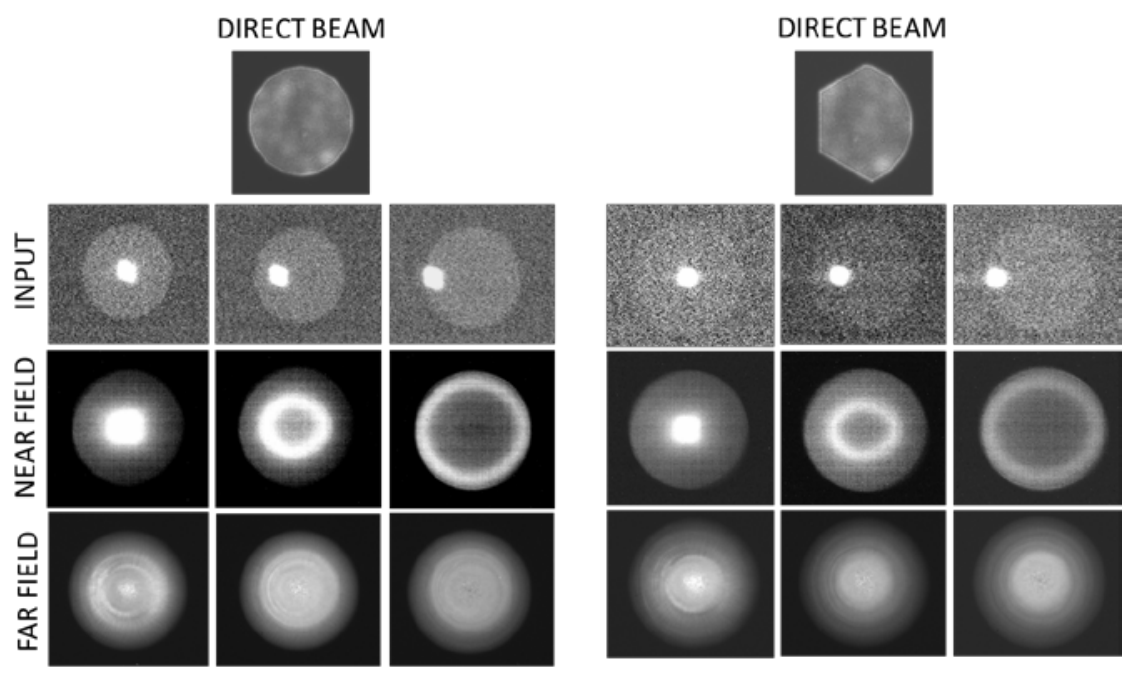

Figure 5. Laboratory measurements of fiber scrambling as a function of pupil shape and spot position. Top row of each configuration shows the direct beam pupil input (left: circular, right: partially obscured hexagonal); $2^{\text {nd }}$ row is a spot imaged onto the fiber input face (center, middle, edge); $3^{\text {rd }}$ and $4^{\text {th }}$ rows are near and far field fiber output. The grating is illuminated by the far field. For typical SALT image quality a star's image will fill the fiber face, resulting in an effective summation of all spot positions.

\section{Spatially resolved near infrared science}

The NIR spectrum contains many useful features for constraining stellar populations in galaxies [5] and has the additional advantage that spectral features here are less affected by dust. The $\lambda=1550-1700 \mathrm{~nm}$ region that we will gain access to with the fiber fed RSS-NIR was specifically targeted by the SDSS-IV APOGEE survey to study the star formation and chemical enrichment history of the Milky Way. For nearby galaxies, this region contains CO bandheads at $\lambda \sim 1620-1630 \mathrm{~nm}$ that indicate the presence of very young $7-10 \mathrm{Myr}$ stars [6].

This spectral region coupled with NIR IFUs can distinguish compact regions of supernova shock-excited gas from diffuse extended regions associated with galactic winds, both of which show up as [Fe II]1644nm emission. Figure 7 shows an example in which [Fe II]1644nm and H $\mathrm{Br}(1588,1611,1641 \mathrm{~nm})$ lines were used to age date spatially distinct bursts of star formation in a galaxy. The $\mathrm{H} \mathrm{Br}$ lines are from recombination of ionized gas in $\mathrm{H} \mathrm{II} \mathrm{regions} \mathrm{that} \mathrm{fades} \mathrm{after}$ $\sim 8 \mathrm{Myr}$, while the [Fe II] emission remains observable for $~ 35-55 \mathrm{Myr}$, placing a lower limit on the time since the star formation was induced [6].

The stellar initial mass function (IMF) can also be constrained by features in the NIR [7]. Different IMFs in stellar population synthesis models change the derived M/L by factors of 3 4, so independent constraints on it are important. The Wing-Ford band, $\mathrm{FeH}$ at $\lambda=991.5 \mathrm{~nm}$, 
and the $\mathrm{Ca}$ I line at $\lambda=1035 \mathrm{~nm}$, which is insensitive to $\alpha / \mathrm{Fe}$ abundance ratio, are particularly good for constraining the low mass end of the IMF.
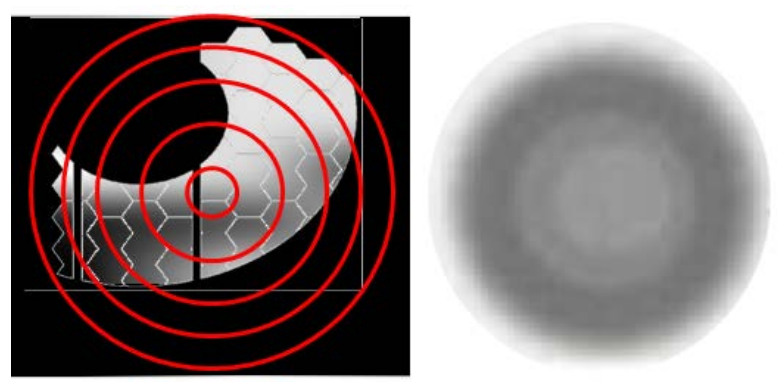

Figure 6. Simulated azmuthal fiber scrambling. Annular rings of modeled SALT pupil images (left) are averaged, smoothed by a gaussian, and summed to form the simulated fiber output image on the right.

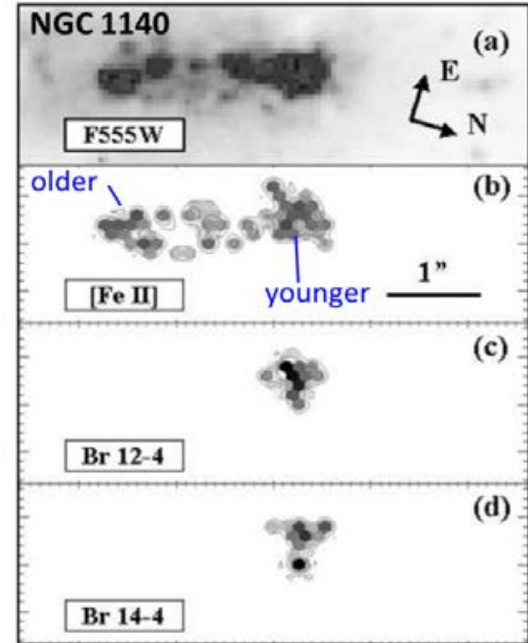

Figure 7. IFU observations from the CIRPASS IFU on Gemini [6].

Large optical IFU galaxy surveys like MaNGA are revealing the ubiquity of extraplanar diffuse ionized gas in galaxies. The RSS-NIR IFU on SALT will be a powerful tool for followup observations of these regions in nearby galaxies for more detailed studies of shocks, stellar ages, and the stellar IMF. This additional information will enable us to construct more complete galaxy evolution histories.

\section{References}

[1] E.B. Burgh, et al., The Prime Focus Imaging Spectrograph for the Southern African Large Telescope: Optical Design, in proceedings of SPIE Astronomical Telescopes + Instrumentation 2003 (4841) 1463.

[2] M.J. Wolf, et al., Project status of the Robert Stobie spectrograph near infrared instrument (RSSNIR) for SALT, , in proceedings of SPIE Astronomical Telescopes + Instrumentation 2014 (9147) 91470B.

[3] K. Bundy, et al., Overview of the SDSS-IV MaNGA Survey: Mapping Nearby Galaxies at Apaceh Point Observatory, ApJ 2015 (708) 7.

[4] A.D. Eigenbrot, et al., The impact of surface-polish on the angular and wavelength dependence of fiber focal ratio degradation, in proceedings of SPIE Astronomical Telescopes + Instrumentation 2012 (8446) 84465W.

[5] J.T. Rayner, et al., The Infrared Telescope Facility (IRTF) Spectral Library: Cool Stars, ApJS 2009 (185) 289.

[6] R. de Grijs, et al., CIRPASS near-infrared integral-field spectroscopy of massive star clusters in the starburst galaxy NGC 1140, MNRAS 2004 (352) 263.

[7] R.J. Smith, et al., The stellar initial mass function in red-sequence galaxies: 1- $\mu$ m spectroscopy of Coma cluster galaxies with Subaru/FMOS, MNRAS 2012 (426) 2994. 